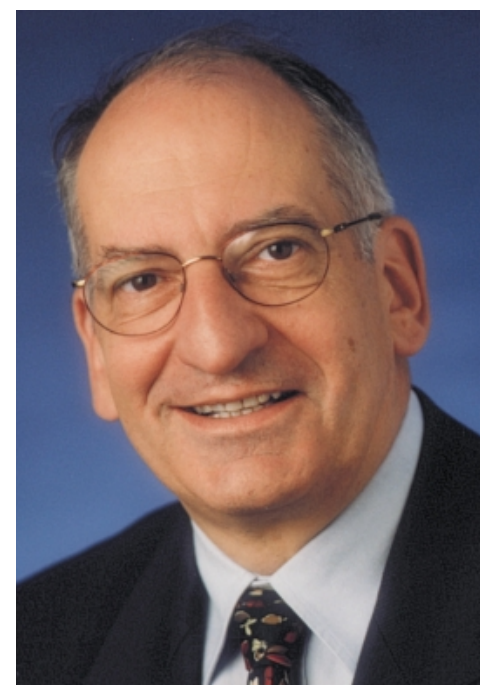

\title{
Die schweizerische Ärzteschaft ist durch die Abkommen nicht bedroht!
}

\author{
Bundesrat Pascal Couchepin, Vorsteher des EVD
}

Die Bedeutung der bilateralen Abkommen für die Schweiz kann nicht hoch genug eingeschätzt werden. Fast ein Jahrzehnt nach der Schaffung des europäischen Binnenmarktes und des schweizerischen Neins zum EWR sichern die Abkommen der Schweiz den weitgehend ungehinderten Zutritt zu diesem für unser Land und unsere Wirtschaft existentiellen Markt. Die Bedeutung der Abkommen liegt auch im psychologischen Bereich: Mehr als ein Vierteljahrhundert nach dem Abschluss des Freihandelsabkommens mit der Europäischen Wirtschaftsgemeinschaft (1972) ist es Zeit, dass wir einen Schritt in Richtung Normalisierung machen, so bescheiden dieser Schritt in Anbetracht der gewaltigen Fortschritte des europäischen Einigungsprozesses auch sein mag. Mit den bilateralen Verträgen schütten wir zwar nicht die Gräben zu, die uns vom Binnenmarkt trennen. Wir bauen jedoch mehrere Brücken, die den Zugang zu diesem Markt für Personen, Güter und Dienstleistungen erheblich erleichtern.

Eines der wichtigsten Abkommen dürfte das Abkommen zur Personenfreizügigkeit sein. Dieses erlaubt es den Schweizer Unternehmen, ihrem Bedarf an qualifizierten Arbeitskräften nachzukommen - wovon die ganze Volkswirtschaft profitiert -, und es ermöglicht insbesondere unseren jüngeren Bürgerinnen und Bürgern, Berufserfahrung im europäischen Ausland zu sammeln. Zugleich ist es ein auf Schweizer Bedürfnisse und Bedenken massgeschneiderter Lösungsweg, der ein bedächtigeres Öffnungstempo erlaubt und mit zahlreichen Sicherheiten versehen ist.

Verhandlungen sind immer auch ein Geben und ein Nehmen. Natürlich hat die Schweiz im Interesse des Ganzen Kompromisse eingehen müssen: Die Verträge bilden zusammen ein Paket, das nur gesamthaft in Kraft (und ausser Kraft) gesetzt werden kann. Es ist nicht möglich nur die Rosinen, beispielsweise das für unsere Fluggesellschaften sehr wichtige $\mathrm{Ab}-$ kommen über die Freiheiten im Luftverkehr oder das von der Pharma- und Maschinenindustrie dringend gewünschte Abkommen zum Abbau technischer Handelshemmnisse herauszupflücken. Der Bundesrat ist aber überzeugt, dass aus dem Blickwinkel der Gesamtwohls unseres Landes die Bilanz klar positiv ist.

Auch für Ärzte und Medizinalpersonal ist diese Marktöffnung grundsätzlich positiv. Sie bietet Chancen für Schweizer Ärzte in der EU und kann eine bessere Versorgung mit medizinischen Leistungen in der Schweiz bringen, insbesondere in einigen Bereichen der Spezialmedizin.

Ich weiss, dass diese Öffnung unter der Ärzteschaft zu Diskussionen Anlass gibt, und ich verstehe die Befürchtungen der Schweizer Ärzte. Trotzdem stellen die Abkommen keine Bedrohung des schweizerischen Ärztestandes oder irgendeines anderen Berufsstandes dar. Aufgrund der im internationalen Vergleich sehr hohen Ärztedichte und den beträchtlichen Investitionen für eine Praxiseröffnung in der Schweiz ist kein massiver Zustrom von Ärzten aus Deutschland, Österreich, Frankreich oder Italien in die Schweiz zu erwarten. Sicherlich ist es möglich, dass einige von den schätzungsweise 2300 an Schweizer Spitälern arbeitenden EU-Ärzten (dies entspricht etwa 8 Prozent der gesamten Ärzteschaft in der Schweiz) die neuen beruflichen Möglichkeiten nutzen werden. Es kann daher vorübergehend zu einer etwas höheren Anzahl von Praxiseröffnungen kommen. Dies stellt aber ein Problem in einer Übergangsphase dar und sollte auf die langfristige Entwicklung der Ärztezahlen keine spürbaren Auswirkungen haben. Dies bestätigen Erfahrungen in der EU, insbesondere in Österreich. Sollte wider Erwarten die Zahl der niedergelassenen Ärzte stark zunehmen, so verfügt der Bundesrat über die notwendigen Instrumente, die Zulassung von Leistungserbringern zur Tätigkeit zu Lasten der obligatorischen Krankenpflegeversicherung einzuschränken (Beschluss des Parlaments vom März dieses Jahres). Ich bin aber überzeugt, dass die hervorragende Qualität der Dienstleistungen der Schweizer Ärzte und das Vertrauen der Patienten, das unsere Ärzte dadurch zu Recht geniessen, sich auch gegenüber einer allfälligen verstärkten Konkurrenz durchsetzen wird. 